\title{
On the sum of the r-th powers of the first $n$ integers
}

\section{By A. Waterson.}

In this note an explicit expression is obtained for the sum of the $r$-th powers of the first $n$ integers. The result is equivalent to the well-known result in terms of Bernoulli numbers and the equivalence is not difficult to establish. However, the method given here is elementary and self-contained and provides an excellent exercise on the manipulation of determinants.

Throughout the note, the following notation will be used:

$$
\begin{aligned}
& S_{n}^{r} \equiv 1^{r}+2^{r}+\ldots+n^{r}, \\
& \left(\begin{array}{l}
n \\
r
\end{array}\right) \equiv n(n-1)(n-2) \ldots(n-r+1) / r ! .
\end{aligned}
$$

We begin with the identities

$$
\begin{aligned}
& (n+1)^{r+1}-n^{r+1}=1+\left(\begin{array}{c}
r+1 \\
1
\end{array}\right) n+\left(\begin{array}{c}
r+1 \\
2
\end{array}\right) n^{2}+\ldots \ldots+\left(\begin{array}{c}
r+1 \\
r
\end{array}\right) n^{r}, \\
& n^{r+1}-(n-1)^{r+1}=1+\left(\begin{array}{c}
r+1 \\
1
\end{array}\right)(n-1)+\left(\begin{array}{c}
r+1 \\
2
\end{array}\right)(n-1)^{2}+\ldots \\
& +\left(\begin{array}{c}
r+1 \\
r
\end{array}\right)(n-1)^{r} \\
& 2^{r+1}-1^{r+1}=1+\left(\begin{array}{c}
r+1 \\
1
\end{array}\right) 1+\left(\begin{array}{c}
r+1 \\
2
\end{array}\right) 1^{2}+\ldots+\left(\begin{array}{c}
r+1 \\
r
\end{array}\right) 1^{r}
\end{aligned}
$$

and obtain, by addition, the identity

$$
(n+1)^{r+1}-1=S_{n}^{0}+\left(\begin{array}{c}
r+1 \\
1
\end{array}\right) S_{n}^{1}+\left(\begin{array}{c}
r+1 \\
2
\end{array}\right) S_{n}^{2}+\ldots\left(\begin{array}{c}
r+1 \\
r
\end{array}\right) S_{n}^{r} \text {. }
$$

Rewriting this result for the values of $r$ from 1 to $r$, we obtain

$$
\begin{aligned}
& (n+1)^{r+1}-1=S_{n}^{0}+\left(\begin{array}{c}
r+1 \\
1
\end{array}\right) S_{n}^{1}+\left(\begin{array}{c}
r+1 \\
2
\end{array}\right) S_{n}^{2}+\ldots\left(\begin{array}{c}
r+1 \\
r
\end{array}\right) S_{n}^{r} \text {, } \\
& (n+1)^{r}-1=S_{n}^{0}+\left(\begin{array}{l}
r \\
1
\end{array}\right) S_{n}^{1}+\left(\begin{array}{l}
r \\
2
\end{array}\right) S_{n}^{2}+\ldots+\left(\begin{array}{c}
r \\
r-1
\end{array}\right) S_{n}^{r-1}, \\
& (n+1)^{2}-1=S_{n}^{0}+\left(\begin{array}{l}
2 \\
1
\end{array}\right) S_{n}^{1}, \\
& (n+1)^{1}-1=S_{n}^{0} \text {. }
\end{aligned}
$$


These equations may be regarded as a set of $(r+1)$ linear equations in the $(r+1)$ unknowns $S_{n}^{0}, S_{n}^{1}, \ldots, S_{n}^{r}$, whence by Cramer's Rule

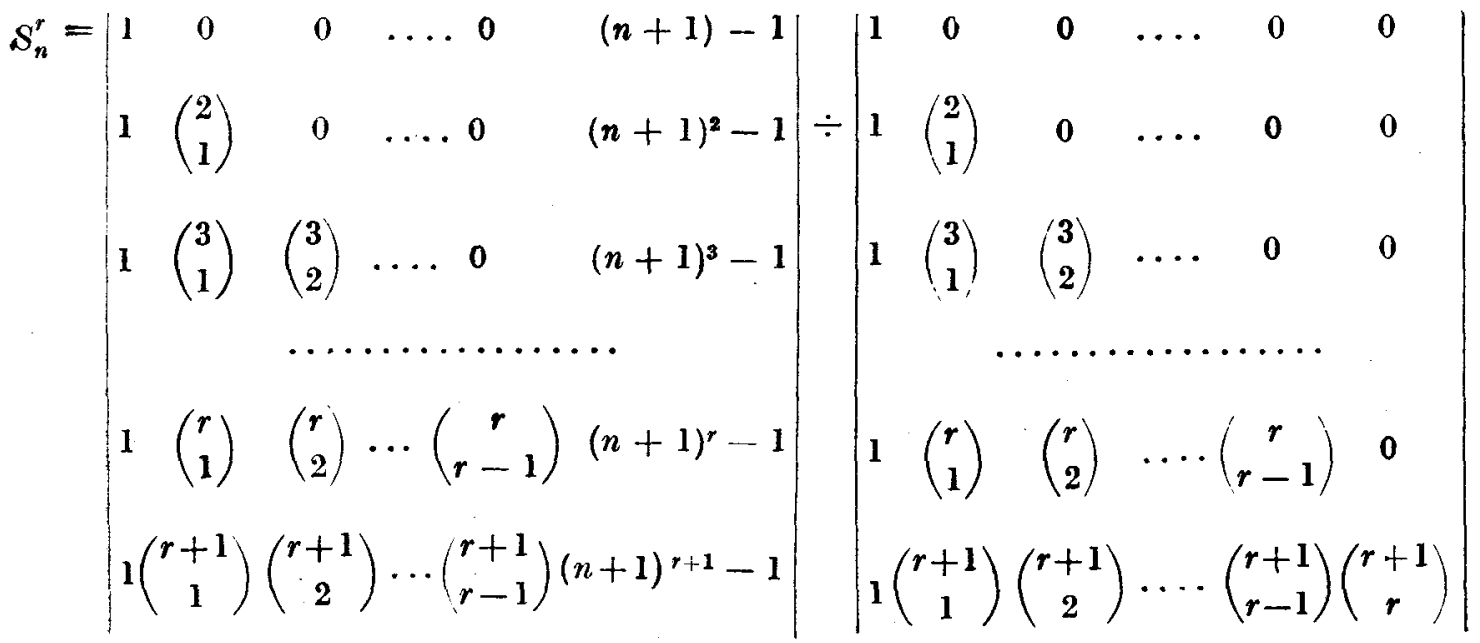

or, since the divisor has the value $(r+1)$ !,

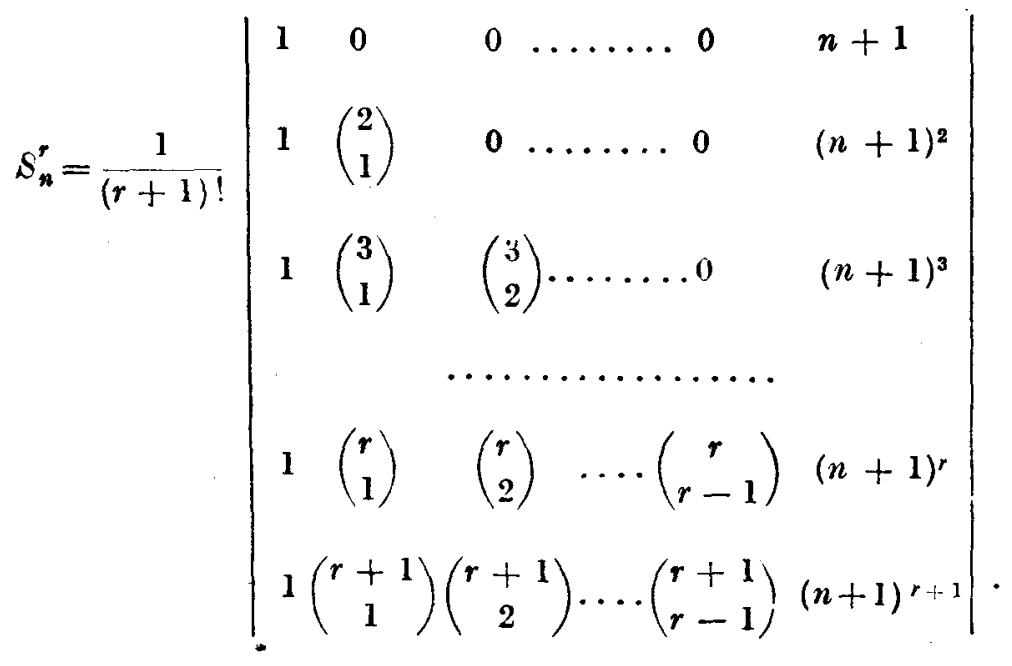

By subtracting from the last column of this determinant the first column, $n$ times the second column, $n^{2}$ times the third column and so on, we find that 


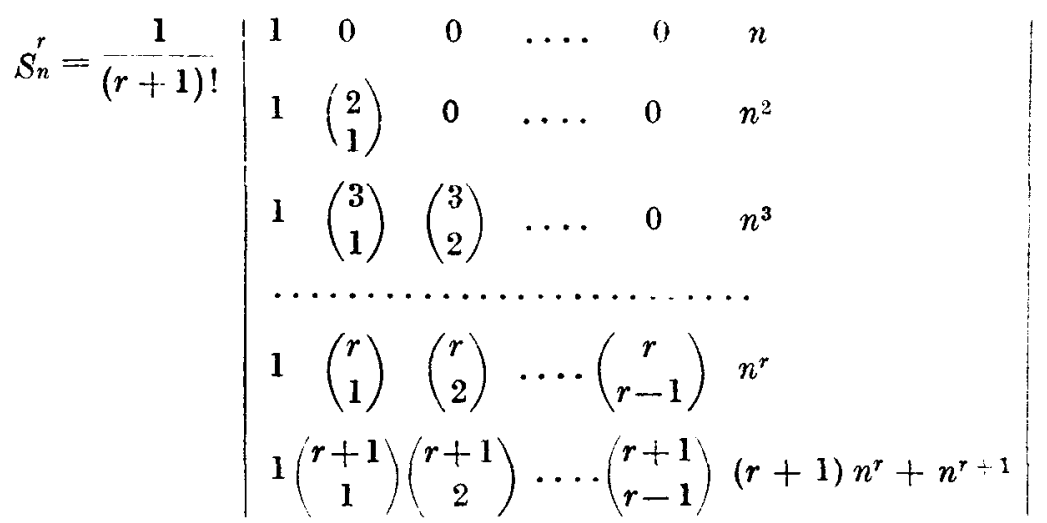

Since the cofactor of the element $(r+1) n^{r}+n^{r+1}$ has the value $r$ ! and that of $n^{r}$ has the value $-\left(\begin{array}{l}r+1 \\ r-1\end{array}\right)(r-1)$ !, i.e. $-\frac{1}{2}(r+1)$ !, the coefficient of $n^{r}$ in the expansion of the determinant is $\frac{1}{2}(r+1)$ ! and (1) may be written in the rather more compact form

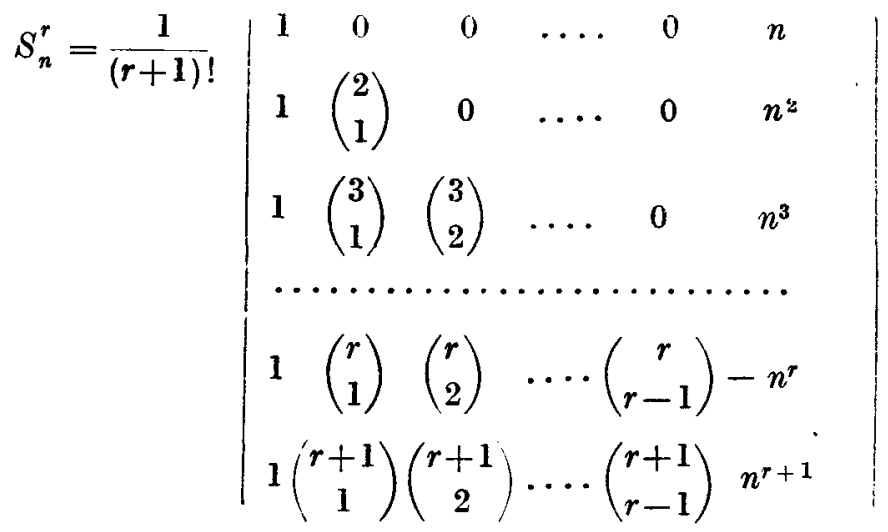

By taking out appropriate factors from the rows and columns of this determinant, we find that

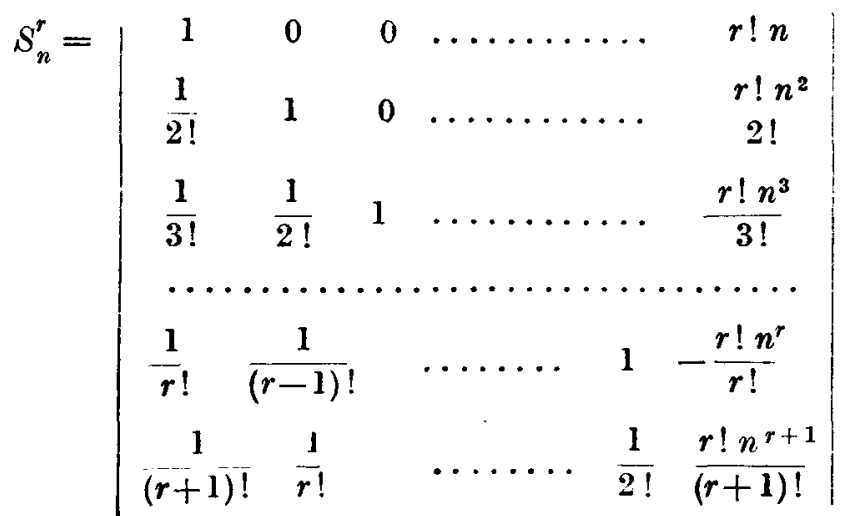


whence, expanding in terms of the cofactors of the elements of the last column, we obtain

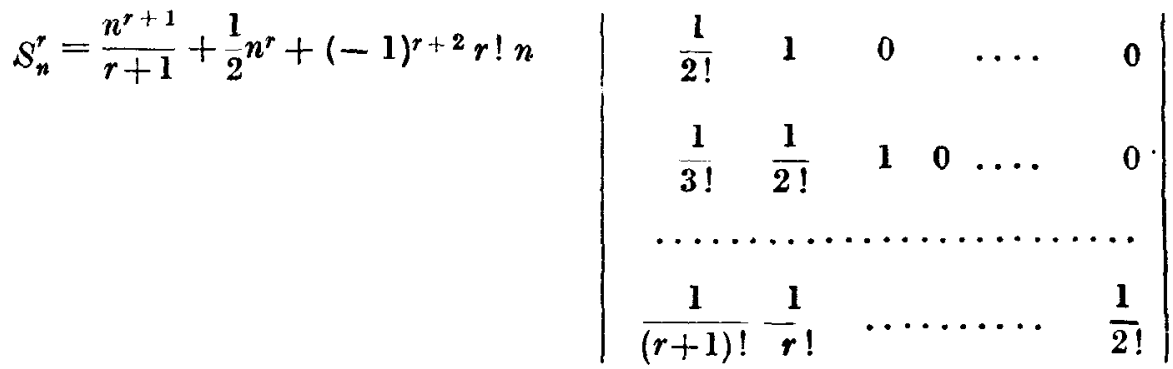

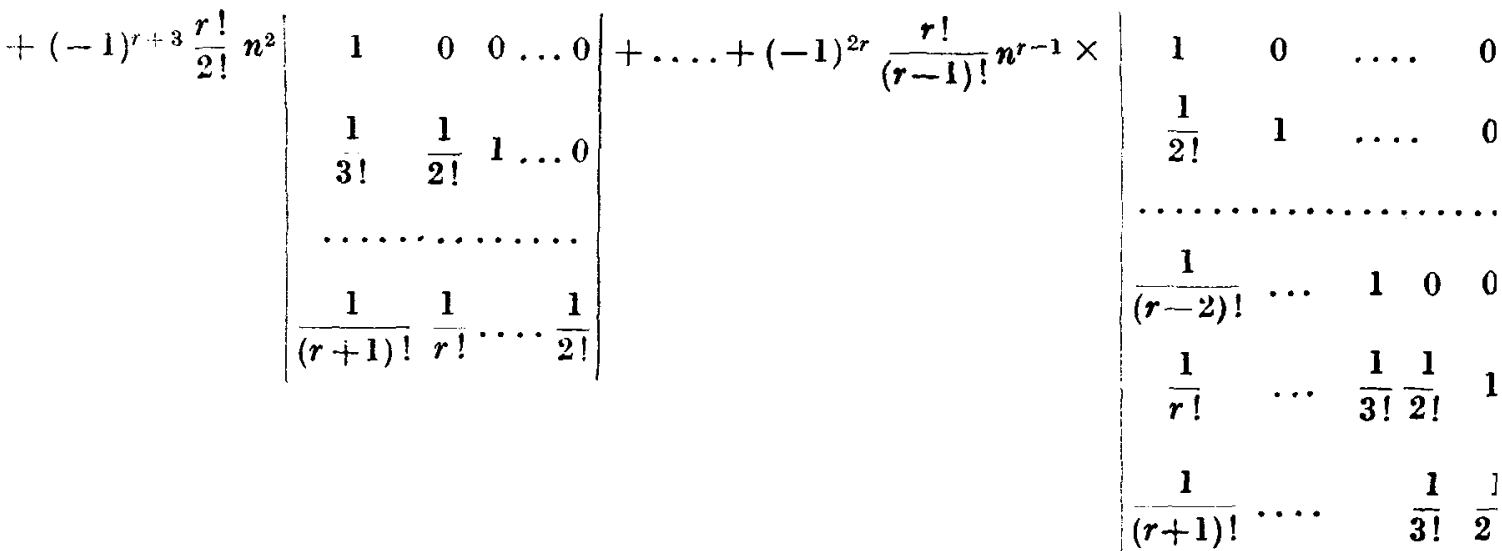

The determinants here can be simplified further and the result written in the form

$$
\begin{aligned}
& \left.S_{n}^{r}=\frac{n^{r+1}}{r+1}+\frac{1}{2} n^{r}+i-1\right)^{r+2} r ! n\left|\begin{array}{ccccc}
\frac{1}{2 !} & 1 & 0 & \ldots & 0 \\
\frac{1}{3 !} & \frac{1}{2 !} & 1 & \ldots & 0 \\
\ldots & \ldots & \ldots & \ldots & \ldots \\
\frac{1}{(r+1) !} & \ldots & \ldots & \frac{1}{3 !} & \frac{1}{2 !}
\end{array}\right| \\
& +(-1)^{r+3} \frac{r !}{2 !} n^{2}\left|\begin{array}{ccccc}
\frac{1}{2 !} & 1 & 0 & \ldots & 0 \\
\vdots & & & &
\end{array}\right|+\ldots+\frac{r !}{(r-1) !} n^{r-1}\left|\begin{array}{cc}
\frac{1}{2 !} & 1 \\
\frac{1}{3 !} & \frac{1}{2 !}
\end{array}\right| . \\
& \frac{1}{r !} \ldots \ldots \frac{1}{2 !}
\end{aligned}
$$


i.e. $S_{n}^{r}=\frac{n^{r+1}}{r+1}+\frac{1}{2} n^{r}+\sum_{k=1}^{r-1}(-1)^{k+1} \frac{r !}{(r-k) !} n^{r-k}\left|\begin{array}{ccccc}\frac{1}{2 !} & 1 & 0 & \ldots & 0 \\ \frac{1}{3 !} & \frac{1}{2 !} & 1 & \ldots & 0 \\ \ldots & \ldots & \ldots & \ldots & \ldots \\ \ldots & \ldots & \ldots & \ldots & 1 \\ 2 ! & 1 \\ \frac{1}{(k+2) !} & \ldots & & \frac{1}{3 !} & \frac{1}{2 !}\end{array}\right|$

This is the desired result, although in some respects the equivalent result (2) is preferable to (3), for apart from the final elements, the successive rows (except the last) are merely those of the well-known Arithmetical Triangle without the final 1 in each case.

Arakan,

134 Halbeath Road, DUNFERMLine.

\section{Inequalities for solutions of linear differential equations A contribution to the theory of servomechanisms}

By HaNs B̈̈̈rNer.

§1. Introduction.

Consider the $n^{\text {th }}$ order differential equation

$$
y+c_{1} y^{\prime}+c_{2} y^{\prime \prime}+\ldots+c_{n} y^{(n)}=f(x),
$$

where the coefficients $c_{v}$ are real constants and $f$ is a real function continuous in the interval $a \leqq x \leqq b$. The following theorem will be proved in $\$ 4$ :

If the characteristic equation of (1) has no purely imaginary roots, then a particular integral $\eta(x)$ can always be found which satisfies the inequality

$$
|\eta| \leqq C M,
$$

where $C$ is a certain function of the $c_{\nu}$ only and $M$ is the maximum of $|f|$. In particular we may take $C=1$ if all roots of the characteristic equation are real. 